\title{
On Legal Suggestions of China's Countermeasures against
}

\section{Surrogacy}

\section{Liu CQ* \\ Professor of Law Institute, Shanghai Academy of social Sciences, China}

*Corresponding author: Chang Qiu Liu, Professor of Law Institute, Shanghai Academy of social Sciences. Majoring in bioethics and law. No. 7, Lane 622, Middle Huaihai road, Shanghai, Post code: 200020, China, Email: shangujushi@sina.com

\section{Opinion \\ Volume 2 Issue 1}

Received Date: December 20, 2019

Published Date: December 31, 2019

DOI: $10.23880 /$ abca- 16000112

\section{Abstract}

China should definitely prohibit surrogacy in law for at least three reasons. First, surrogacy is a serious violation of human nature which will hurt maternity. Second, surrogacy is a risky business that can do genuine harm to a woman's body. Third, legalizing surrogacy is not a rational response to the present demand for surrogate mothers within China. There are some defects at present in China's law against surrogacy, which need rectification.

Keywords: Surrogacy; China; Law; Bioethics

\section{Opinion}

In recent years, surrogacy has been a hot word in China as so many news about surrogacy have been reported by the medium. With the development of human assisted reproductive technology while more and more people can't bear their babies by themselves, surrogacy has been an optional for many people to have a baby of their own. More and more scholars suggest that Chinese government should release the ban of surrogacy so as to make it legalized. But I am wholly against any attempt to legalize surrogacy in China. In fact, I think legal prohibitions against surrogacy should be strengthened for three main reasons.

First, surrogacy is inhuman. It is a serious violation of human nature which will hurt maternity. It is well-known in medical and psychological literature that over the course of a pregnancy, a surrogate mother naturally develops an attachment to the child growing inside her.
This occurs regardless of her lack of shared genetic material and has more to do with the way the fetus's bodily systems grow and intertwine with those of the woman carrying it. To then force the woman to hand over that child, to entrust the baby to someone else, is a serious violation of her maternal instincts. Loosening restrictions on surrogacy is no different from encouraging women to abandon, or at least ignore, what their bodies naturally tell them to do.

Second, surrogacy is a risky business that can do genuine harm to a woman's body. In practice, surrogates risk their lives and health, not to mention the possibility of postpartum depression, weight problems, and the pain of childbirth-all so other people can have a child. Having a baby puts immense stress on the body and mind, and such trauma runs counter to the best interests of the surrogate herself. 
Finally, legalizing surrogacy is not a rational response to the present demand for surrogate mothers within China. There are hundreds of thousands of women who can have their babies only by surrogacy, while there are little women who are reluctant to be a surrogate mother without pay. So only lifting restrictions on commercial surrogacy might possibly meet this demand, because the vast majority of women will only take on the risks of surrogacy if offered some kind of financial incentive.

Commercial surrogacy is currently illegal in a significant number of the world's developed countries. Some Chinese scholars advocating that we legalize surrogacy almost universally throw their weight behind what is known as altruistic surrogacy, in which the mother receives no compensation beyond reimbursement for medical and other expenses. These advocates would, however, maintain the ban on commercial surrogacy.

In reality, the legalization of altruistic surrogacy will not increase the number of available mothers. Women are the best judges of their own interests and are therefore unlikely to take on the role of a surrogate without adequate financial compensation. While some may ultimately decide to do it anyway, they will represent only a small minority of women. In essence, then, legalizing altruistic surrogacy is a wholly inadequate method of making up for the shortage of willing surrogates. I believe that surrogacy is only a life-saving straw, not a lifeboat. Even if it is a life boat. How many people could it hold? Ten, twenty? But outside of the boat, there are millions. In a word, surrogacy is not the right way for the more and more women to solve the problems not to have a baby of their own.

In the past, China paid more and more attention to the construction of bioethics to play the role in deal with biotechnology activities, while the countermeasures against surrogacy appear always ineffective. The step of enactment of law is very slow. And the enforcing legal document on surrogacy, which has prohibited medical institutions and doctors to offer surrogacy service to the patients, is only a regulation enacted by the ministry of public health which has given and can give little punishment to surrogacy. What's more, criminal punishment couldn't be stipulated in this regulation. Thus makes more and more surrogacy services be offered without scruple by some indecent medical institutions as well as doctors. Many surrogate babies have been born. In the future, China should speed up to enact effective law on human assisted reproductive technology to prohibit surrogacy strictly. What's more, as far as I am concerned, it's very necessary for China to revise its Criminal Law to regard surrogacy as crimes since surrogacy has been to a deluge, which has done great harm to the traditional values of Chinese society. More so, China should deal seriously with the protection of the surrogate babies in its civil law as more and more surrogate babies have been born through illegal surrogacy. The principle of the best interest for children should be fixed in civil law. It is only under such measures that surrogacy might be controlled to the level that the society may endure. 